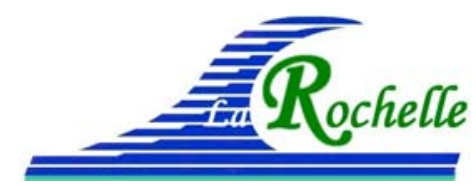

XVèmes Journées Nationales Génie Côtier - Génie Civil

La Rochelle, 29 au 31 mai 2018

DOI:10.5150/jngcgc.2018.004 ～(C) Editions Paralia CFL

disponible en ligne - http://www.paralia.fr - available online

\title{
Modélisation des surcotes avec HYCOM pour les départements d'outre-mer
}

\section{Sophie CASITAS ${ }^{1}$, Audrey PASQUET ${ }^{1}$, Héloïse MICHAUd ${ }^{1}$, Rémy BARAILLE ${ }^{1}$, Didier JOURDAN ${ }^{1}$}

\begin{abstract}
1. Shom - Météopole, 42 avenue G. Coriolis, BP 63576, F-31035 Toulouse, France. sophie.casitas@shom.fr
\end{abstract}

\section{Résumé :}

Le projet HOMONIM (Historique, Observation, MOdélisation des NIveaux Marins) est un projet commun du SHOM et de Météo-France, dont l'objectif est l'amélioration du système français de vigilance et d'alerte vagues-submersions, opéré par Météo-France sur les côtes métropolitaines et les départements d'outre-mer. Ce système combine les capacités de prévisions du modèle océanique HYCOM (BARAILLE \& FILATOFF, 1995) dans un formalisme barotrope, et du modèle d'état de mer WaveWatch $3^{\circledR}$ - WW3 (TOLMAN, 2016).

Depuis 2017, deux nouvelles configurations ont été développées, implémentées et opérées sur les territoires d'outre-mer :

- Pour la configuration Antilles-Guyane, une grille curvilinéaire est utilisée pour atteindre une résolution de $2.5 \mathrm{~km}$ près des côtes de Guyane et de $900 \mathrm{~m}$ autour des iles caribéennes.

- Pour la configuration Sud-Ouest Océan Indien, une grille régulière de $3.2 \mathrm{~km}$ est présentée. Des configurations imbriquées, atteignant une résolution d'une centaine de mètres autour des iles françaises de Mayotte et La Réunion, sont en cours de développement. Dans cette future version, les échanges entre les grilles HYCOM sont réalisés avec le coupleur Oasis-MCT.

Ces systèmes bénéficient d'optimisations numériques et développements physiques tels qu'un nouveau solveur barotrope, une friction sur le fond optimisée par une approche stochastique et des mises à jour des MNT.

Ces deux configurations ainsi que leurs résultats sur quelques évènements tempétueux seront présentés. La sensibilité du modèle à la paramétrisation du stress de vent sera discutée.

Mots-clés :

HYCOM, Surcote, Modélisation des surcotes, Hydrodynamique côtière, Submersion, Risques côtiers, Outre-mer.

\section{Abstract:}

The HOMONIM project (History, Observation and Modeling of sea levels) is a French collaborative effort lead by SHOM and Meteo-France, that aims at improving the 


\section{Thème 1 - Hydrodynamique côtière}

French waves and surge warning system operated by Meteo-France on the metropolitan and overseas coasts. This system combines the forecasting capacities of the oceanic model HYCOM (BARAILLE \& FILATOFF, 1996) in a barotropic formalism, and the sea state model WaveWatch $3^{\circledR}$ - WW3 (TOLMAN, 2016).

Since 2017, two new configurations have been developed, implemented and operated on French overseas territories:

- For the Antilles Guyana configuration, a curvilinear grid is used to reach a resolution of $2.5 \mathrm{~km}$ near the Guyana coast and $900 \mathrm{~m}$ around Caribbean islands.

- For the south West Indian Ocean configuration, a regional regular grid $(3.2 \mathrm{~km})$ is used. Nested configurations reaching a resolution of a few hundred meters on the French islands Mayotte and La Reunion are currently developed. HYCOM grids exchanges are performed by the Oasis-MCT coupler.

These systems benefit from optimized numerical and physical developments, among them: a new barotropic solver, a bottom friction optimized by a stochastic approach, and an up to date DTM.

These two configurations will be presented, as well as their results on significant storm surge events. The model sensitivity to wind stress parametrization will be discussed.

\section{Keywords:}

HYCOM, Storm surge, Surge modelling, Coastal hydrodynamic, Flooding risks assessment, Coastal hazards, French overseas territories.

\section{Introduction}

Le projet HOMONIM - Historique, Observation, MOdélisation des NIveaux Marins est un projet commun du SHOM et de Météo-France, sous maîtrise d'ouvrage de la Direction Générale pour la Prévention des Risques (DGPR) et de la Direction Générale de la Sécurité Civile et de la Gestion de Crise (DGSCGC). Il participe à l'action de l'Etat, engagée dans le cadre du plan interministériel Submersions Rapides, sur "l'amélioration de la connaissance des aléas et des systèmes de surveillance de prévision, de vigilance et d'alerte", et le "développement progressif et la mise en place opérationnelle de modèles océanographiques côtiers", en se fixant l'objectif d'améliorer les capacités de prévision, au profit de l'anticipation des submersions marines.

La phase-I du projet (2012-2015) a permis d'améliorer le dispositif de Vigilance Vagues-Submersions (VVS) sur les façades métropolitaines en renforçant le réseau d'observations et en dotant Météo-France de deux nouvelles chaînes : une de prévision des surcotes et une de prévision des vagues à la côte.

La phase-II (2016-2019), vise à poursuivre cet effort d'amélioration et à l'étendre aux départements d'outre-mer (Antilles, Guyane, Réunion, Mayotte). Pour répondre à cette dernière problématique, deux chaines opérationnelles ont été mises en place dans l'environnement opérationnel de Météo-France et ont vocation à couvrir respectivement le domaine Antilles-Guyane et le sud-ouest de l'Océan Indien. Ces systèmes sont mis à 


\section{XVèmes Journées Nationales Génie Côtier - Génie Civil \\ La Rochelle, 29 au 31 mai 2018}

jour annuellement. Les deux configurations opérées à ce jour (seconde version AntillesGuyane, première version Indien) sont présentées dans la section 2. Des comparaisons entre les observations et les surcotes modélisées lors d'évènements tempétueux sont présentées dans la section 3 .

\section{Les configurations}

2.1 Configuration Antilles - Guyane

a) Le domaine

Le domaine géographique de la configuration Antilles-Guyane s'étend de $64^{\circ} \mathrm{W}$ à $42^{\circ} \mathrm{W}$ en longitude et de 0 à $20^{\circ} \mathrm{N}$ en latitude.

La grille du domaine est curvilinéaire avec une résolution de l'ordre de $2.3 \mathrm{~km}$ au niveau de la Guyane et d'environ $900 \mathrm{~m}$ au niveau des Antilles (figure 1).

La bathymétrie utilisée est le MNT 500 m réalisée par le SHOM dans le cadre du projet HOMONIM (BISCARA, 2017).

\section{b) Le forçage de marée}

Le forçage de marée utilisé pour forcer le modèle HYCOM est issu du modèle de marée global FES2014. La marée est forcée aux frontières ouvertes du domaine AntillesGuyane en élévation de surface pour 20 composantes: M2, S2, N2, K2, K1, O1, P1, Q1, M4, MS4, 2N2, MU2, T2, NU2, R2, L2, J1, MN4, M3, M6.

\section{c) La carte de friction au fond}

La formulation de la friction est semi-quadratique (Eq. 1) :

$$
d r a g=\frac{C b \times \frac{\|u\|}{2}+C b a r}{\max (H t, 0.1)}
$$

Le coefficient de friction quadratique est calculé par la formulation logarithmique obtenue après intégration de la vitesse sur la colonne d'eau (Eq.2). La longueur de rugosité zo ainsi que la partie linéaire de la friction de fond sont obtenues par une optimisation par méthode stochastique adaptée d'après les travaux de BOUTET (2015).

$$
C b=k \frac{1}{\ln \left(\frac{H t}{z_{0}}-1\right)^{2}}
$$

Avec $k$, la constante de Von Karman.

Dans ce processus itératif, la prédiction de marée pour une quarantaine de marégraphes est utilisée comme référence par rapport aux résultats du modèle pour calculer des scores de marée (amplitude et phase pour les Pleines Mers (PM) et les Basses Mers $(\mathrm{BM})$ ). La fonction coût minimisée est définie comme la somme de l'erreur quadratique moyenne des BM et des PM sur une période de 64 jours couvrant trois périodes de vives eaux et mortes eaux. Pour cette configuration, 64 points de collocalisation ont été 


\section{Thème 1 - Hydrodynamique côtière}

utilisés. Le processus de convergence a été stoppé après 271 itérations (plus d'évolution notable des cartes).

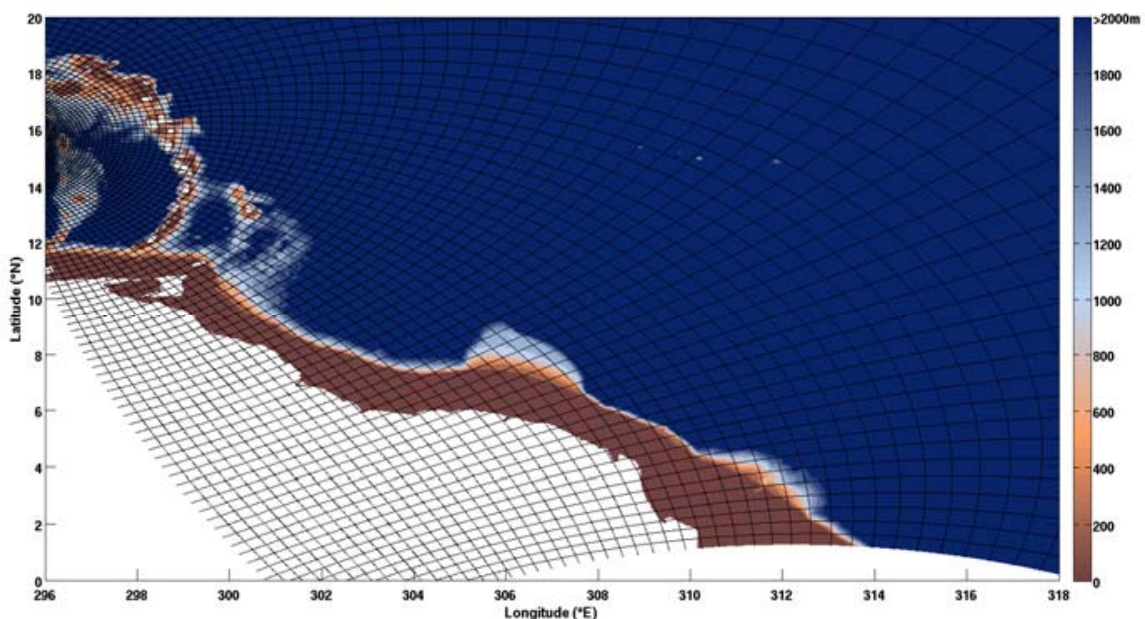

Figure 1. Bathymétrie (m) du domaine Antilles-Guyane issue du MNT 500 m et grille curvilinéaire (1 isoligne sur 20 représentée).

\subsection{Configuration Sud-Ouest Océan Indien}

a) Le domaine

Le domaine modélisé s'étend de 7 à $30^{\circ} \mathrm{S}$ et de 32 à $67.5^{\circ} \mathrm{E}$. La grille de calcul est uniforme avec une résolution d'environ $3.2 \mathrm{~km}$. La bathymétrie utilisée est GEBCO à $30 "$ (figure 2).

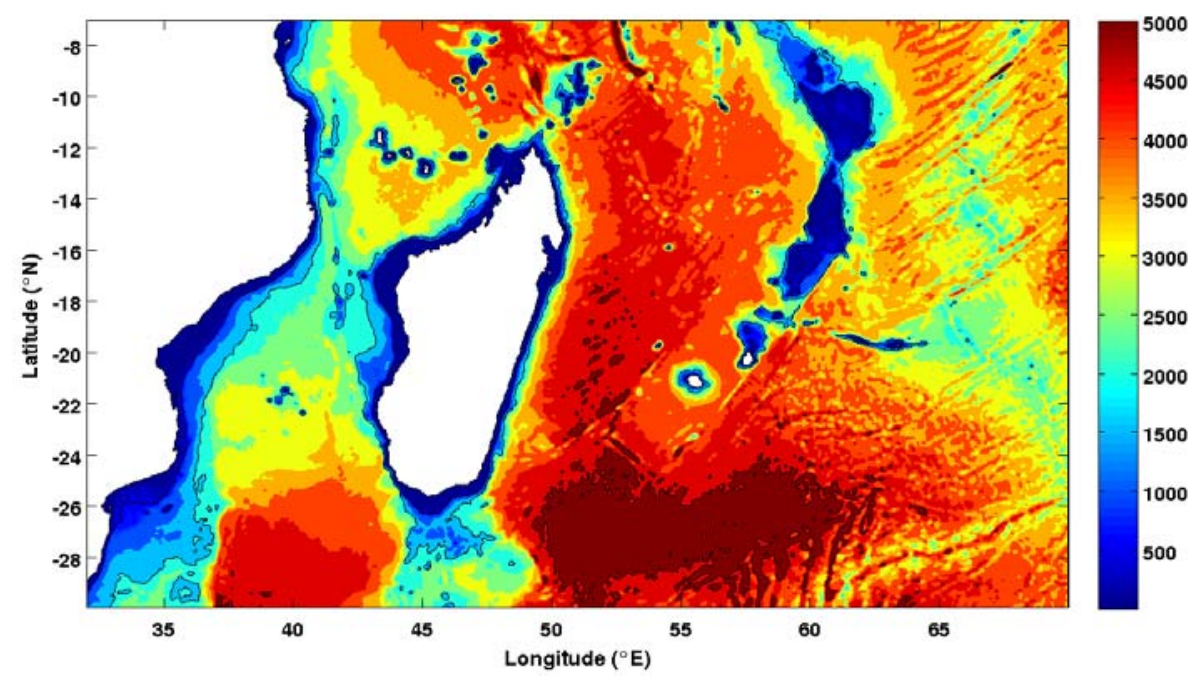

Figure 2. Bathymétrie (m) de la zone INDIEN. Les isobathes 500, 2000 et $5000 \mathrm{~m}$ sont contourées. 


\section{XVèmes Journées Nationales Génie Côtier - Génie Civil \\ La Rochelle, 29 au 31 mai 2018}

\section{b) Le forçage de marée}

Le forçage de marée utilisé pour forcer le modèle HYCOM est issu du modèle de marée global FES2012 au 1/16 ${ }^{\circ}$. La marée est forcée en élévation de surface aux frontières ouvertes du domaine INDIEN pour 20 composantes: M2, S2, N2, K2, K1, O1, P1, Q1, M4, MS4, 2N2, MU2, T2, NU2, R2, L2, J1, MN4, M3, M6.

\section{c) La carte de friction au fond}

Les tests sur le coefficient de frottement sur le fond ont montré que la solution de marée est peu sensible à la friction sur le fond dans les zones à très grandes profondeurs, ce qui est le cas des zones d'intérêt comme Mayotte (hors lagon) et La Réunion aux résolutions considérées. Par contre, l'impact du coefficient de frottement se fait sentir aux endroits de faibles profondeurs, situés essentiellement au niveau du plateau de Beira. Dans cette $1^{\text {ere }}$ configuration mise en place, la friction sur le fond est quadratique avec un $\mathrm{Cb}$ constant pris à $2 \cdot 10^{-3}$.

\section{Les résultats}

Des comparaisons observations / modèle sont réalisées lors d'évènements tempétueux. Les données marégraphiques utilisées pour la comparaison sont en priorité des données fournies par le SHOM aux marégraphes RONIM, quand elles sont disponibles. Dans ce cas, la surcote mesurée a été calculée à partir du niveau d'eau mesuré auquel on a soustrait la hauteur de marée prédite par le SHOM.

Des comparaisons ont également été faites à d'autres marégraphes pour lesquels les données ont été récupérées sur le site de l'IOC (http://www.ioc-sealevelmonitoring.org). Dans ce cas, la surcote mesurée a été calculée à partir de la hauteur observée à laquelle on a soustrait une hauteur prédite calculée après analyse harmonique des séries observées.

\subsection{Configuration Antilles-Guyane}

La représentation de la surcote par le modèle HYCOM est évaluée sur 8 évènements tempétueux dont les cyclones IRMA et MARIA ayant frappé les Antilles en septembre 2017. Les cartes de friction optimisées sont prises en compte pour ces rejeux.

Le stress de vent vent $\tau_{s}$ est calculé par la formulation bulk ci-dessous :

$$
\tau_{s}=\rho_{a} C d U_{10} \quad(E q .3)
$$

Avec $\rho_{a}$ la densité de l'air, U10 l'intensité du vent à $10 \mathrm{~m}$ d'altitude et $\mathrm{Cd}$, la contrainte de surface.

Dans la théorie, Cd peut être constant, ou bien varier en fonction de la vitesse du vent, de l'état de mer,.... 


\section{Thème 1 - Hydrodynamique côtière}

Pour chaque évènement, différentes paramétrisations de la contrainte de surface ont été testées pour tenter d'obtenir un stress de vent qui permette de reproduire au mieux dans le modèle le pic de surcote observé (figure 3):

- La paramétrisation de Pond et Pickard (POND \& PICKARD, 1998): Dans cette paramétrisation, la contrainte de surface varie linéairement avec la vitesse du vent pour des vents faibles ou modérés et elle est seuillée pour des vents supérieurs à $50 \mathrm{~m} / \mathrm{s}: \quad \mathrm{Cd}=0.00061+0.000063 * \max \left(\min \left(\left|\mathrm{u}_{\mathrm{z}}\right|, 50.\right), 6\right.$. $)$ avec $\left|\mathrm{u}_{\mathrm{z}}\right|$, le module de la vitesse du vent.

- La paramétrisation de Charnock (CHARNOCK, 1955): Le coefficient Cd de la paramétrisation de Charnock (1955) est donné par les équations suivantes :

$$
\begin{gathered}
C d=\frac{u_{*}^{2}}{u_{z}^{2}}=k^{2}\left[\ln \frac{z}{z_{0}}\right]^{-2}, z=10 m \\
z_{0=} \alpha_{c} \frac{u_{*}^{2}}{g} \quad \text { (Eq.5) }
\end{gathered}
$$

Avec $z_{0}:$ la longueur de rugosité, $u_{*}:$ la vitesse de friction, $u_{z}:$ la vitesse du vent à la hauteur $\mathrm{z}=10 \mathrm{~m}, \alpha_{c}$ : le paramètre de Charnock constant, $k$ : la constante de Von Karman, $k \sim 0.4$. Dans cette paramétrisation de Charnock constante (indépendante de l'état de mer), $\alpha_{c}$ est le paramètre variable que nous avons testé $\left(\alpha_{c}=0.025\right.$ et 0.015$)$.

- La paramétrisation de Makin (MAKIN, 2005), proche de celle de Charnock, mais pour laquelle la rugosité peut s'écrire :

$$
\mathrm{z}_{0}=\mathrm{C}_{\mathrm{l}}^{(1-1 / \omega)} \alpha_{\mathrm{c}}^{\frac{1}{\omega}} \frac{\mathrm{u}_{*}^{2}}{\mathrm{~g}}(E q .6) \quad \text { et } \quad \omega=\min \left(1, \frac{a_{c r}}{k u_{*}}\right) \quad \text { (Eq.7) }
$$

Avec zo la longueur de rugosité effective prenant en compte l'impact des vagues et des gouttelettes d'eau sur la dynamique de l'écoulement. Cl est une constante dont la valeur a été déduite de mesures (POWELL et al, 2003, $\mathrm{Cl}=10$ ). $\alpha_{\mathrm{cr}}$ est la valeur critique de la vitesse terminale.

- La paramétrisation de Makin modifiée (paramétrisation de Makin dans laquelle on a modifié la pente d'évolution de la contrainte de surface après le point de seuillage de façon empirique afin de reproduire au mieux le pic de surcote observé; $\mathrm{Cl}$ pris à $0.045)$

Pour la majorité des rejeux (tempêtes avec des vitesses de vent "modérées", inférieures à $30 \mathrm{~m} / \mathrm{s}$ ), les 4 paramétrisations citées ci-dessus donnent des résultats équivalents ou très proches. D'autre part, du fait de la faible résolution, des fortes profondeurs et du formalisme 2D, ces effets du vent sont sous-estimés dans le modèle.

Dans le cas d'IRMA avec ses vents extrêmement violents, les contraintes de surface calculées sont très différentes selon les paramétrisations choisies et cela a donc un impact sur les pics de surcotes modélisés. Le choix de la paramétrisation du stress du vent pour cette configuration s'est donc basé sur les résultats des rejeux du cyclone IRMA, pour lesquels des pics de surcotes ont été enregistrés pour 2 ports marégraphiques, à Saint-Martin Marigot et Barbuda (figure 4). 


\section{XVèmes Journées Nationales Génie Côtier - Génie Civil \\ La Rochelle, 29 au 31 mai 2018}

Les graphes mesures / modèle ci-dessous montrent que dans le cas de IRMA, les paramétrisations du stress de vent qui permettent une bonne représentation du pic de surcote avec les forçages fournis par Météo-France (modèle Arome Tropique, simulation du 5/09 à 18h) sont les paramétrisations de Pond et Pickard (1998) et la paramétrisation de Makin modifiée.

L'avance temporelle du pic dans le modèle $(30 \mathrm{mn})$ s'explique par un cyclone en avance dans les forçages utilisés. La figure 5 illustre les résultats du modèle HYCOM pour IRMA en utilisant les forçages du 5/09 18h et les forçages du 06/06 00h. Selon la simulation, le pic de surcotes modélisé est décalé de $30 \mathrm{mn}$ en avance (pour les forçages issus de la simulation du 05/09 18h) / en retard (pour les forçages issus de la simulation du 06/09 00h) par rapport au pic mesuré. Les amplitudes des pics modélisés sont également très différentes $(1.50 \mathrm{~m}$ d'écart). Les résultats du modèle de surcote sont donc extrêmement sensibles aux forçages atmosphériques utilisés et à la trajectoire modélisée des cyclones tropicaux, du fait de la faible étendue du phénomène et des grandes intensités des vents.

Le calcul du stress de vent avec la paramétrisation de Pond et Pickard (cd_PP98), qui nécessite un temps de calcul faible par rapport à la solution d'une paramétrisation de Makin (convergence par processus itératif), semble être un bon compromis pour le calcul du stress de vent pour la configuration Antilles-Guyane.

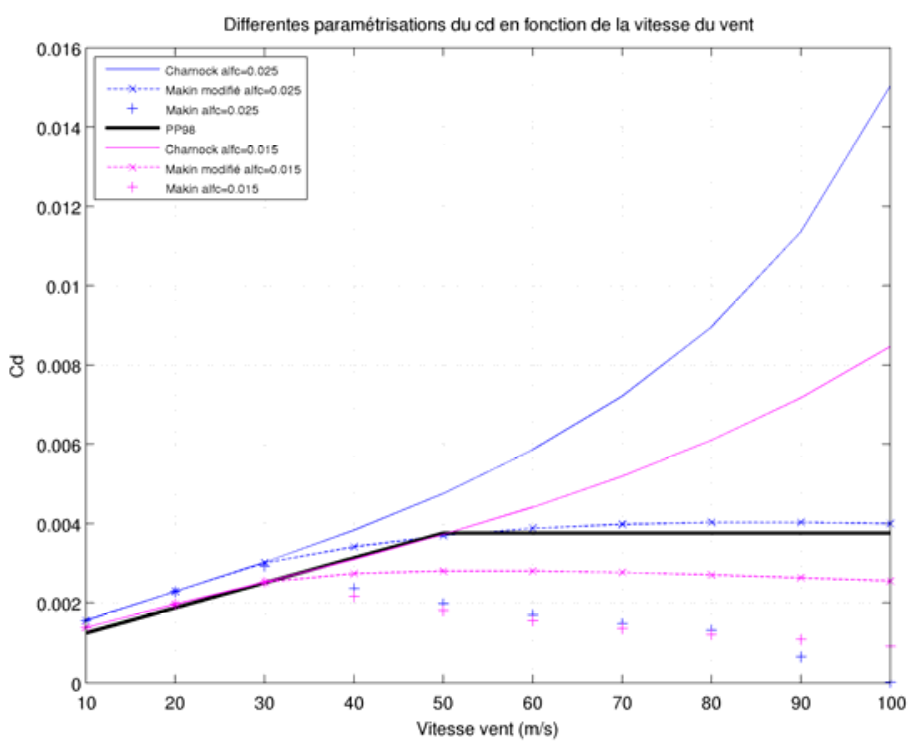

Figure 3. Evolution de la contrainte de surface Cd en fonction de la vitesse du vent, pour une paramétrisation de Charnock constant (en continu), de Makin (+), de Makin modifié (ligne avec marqueurs) pour un coefficient de Charnock $\alpha_{c}$ de 0.025(bleu) et 0.015(rose), et pour une paramétrisation de Pond \& Pickard (1998, en noir). 

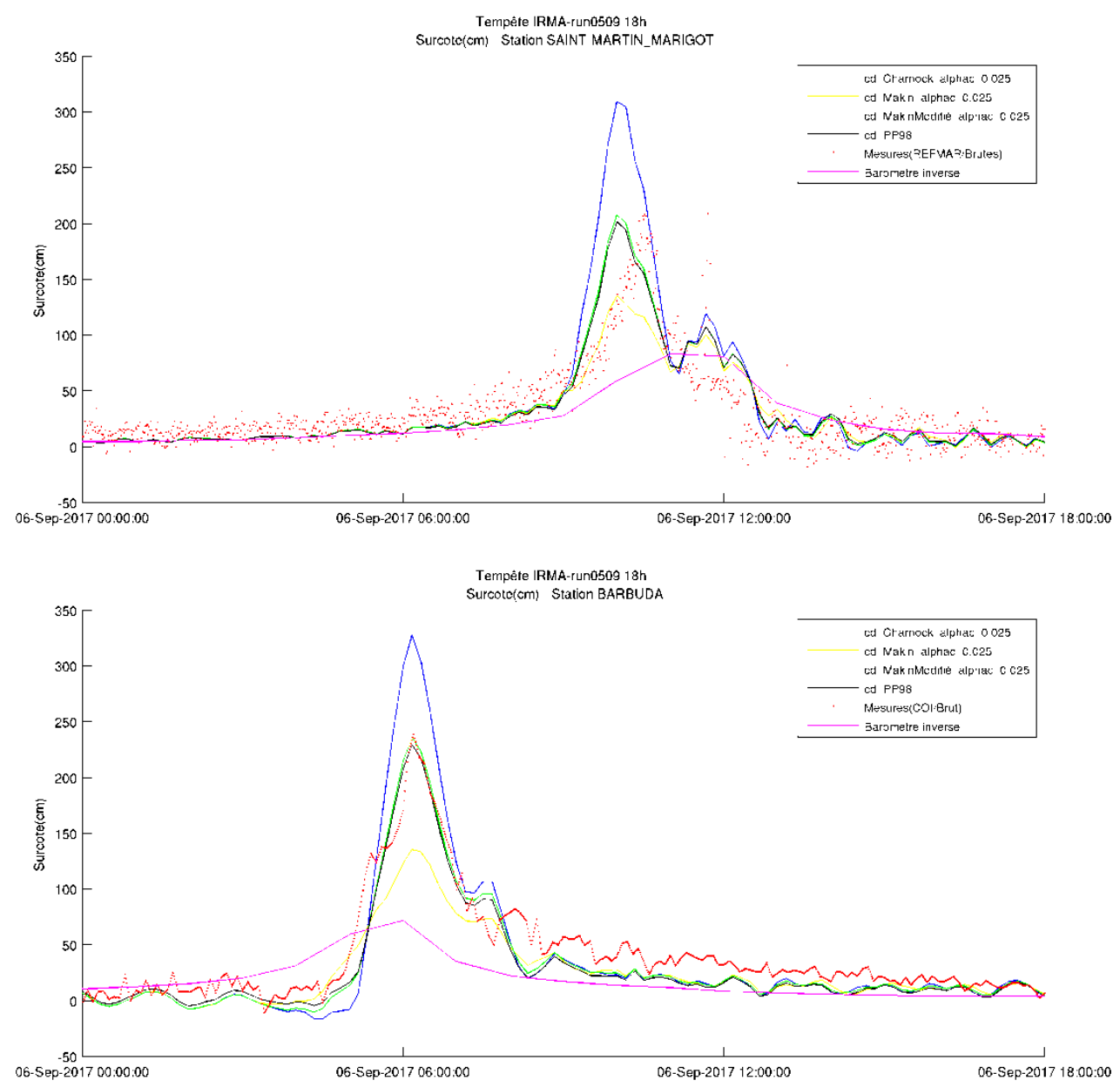

Figure 4. Surcotes observée et modélisées par HYCOM sur la période du 6 et 7 septembre 2017 à Saint-Martin Marigot (en haut) et Barbuda (en bas).

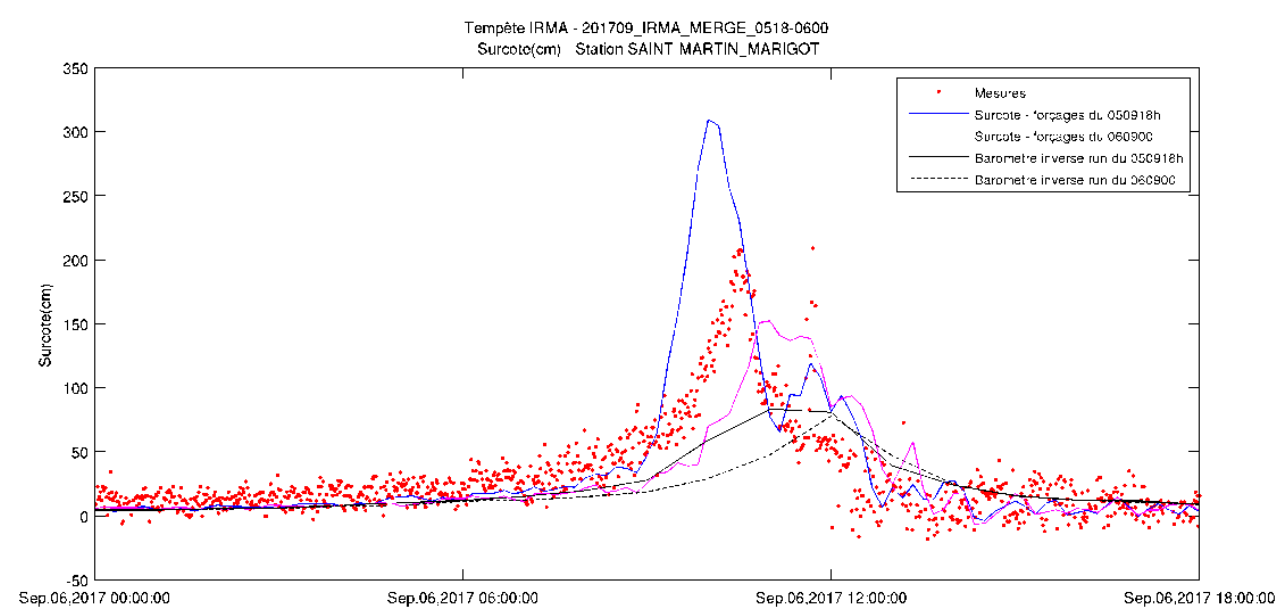

Figure 5. Résultat du modèle HYCOM à Saint-Martin Marigot utilisant les forçages du 05/09 18h (en bleu) et du 06/09 00h (en rose) avec une paramétrisation constante de Charnock $\alpha_{c}=0.025$. 


\section{XVèmes Journées Nationales Génie Côtier - Génie Civil \\ La Rochelle, 29 au 31 mai 2018}

\subsection{Configuration Sud-Ouest Océan Indien}

Le modèle de surcote a été mis en œuvre sur une dizaine d'évènements tempétueux qui ont traversé le domaine INDIEN. Pour chaque tempête, trois paramétrisations de stress de vent ont été testées $\left(\mathrm{Cd}=0.002\right.$; paramétrisation de Charnock constante avec $\alpha_{c}=$ 0.015 et 0.025 ).

Des comparaisons observations aux marégraphes / modèle à Pointe des Galets (Réunion) pour 2 évènements sont présentées ci-dessous.

Les tests de sensibilité montrent que la paramétrisation du stress de vent n'a pas d'impact significatif sur les hauteurs des surcotes générées lors du passage des tempêtes. Pour chaque cas, la surcote modélisée est très proche de la surcote barométrique (voir courbe noire sur la figure 6). La sous-estimation par rapport aux mesures suggère une mauvaise prise en compte des effets du vent. La décote après le pic, qui n'est pas reproduite dans le modèle, pourrait également être attribuable au formalisme barotrope (sous-estimation de l'effet $\mathrm{du}$ vent, dynamique $3 \mathrm{~d}$ non reproduite...)
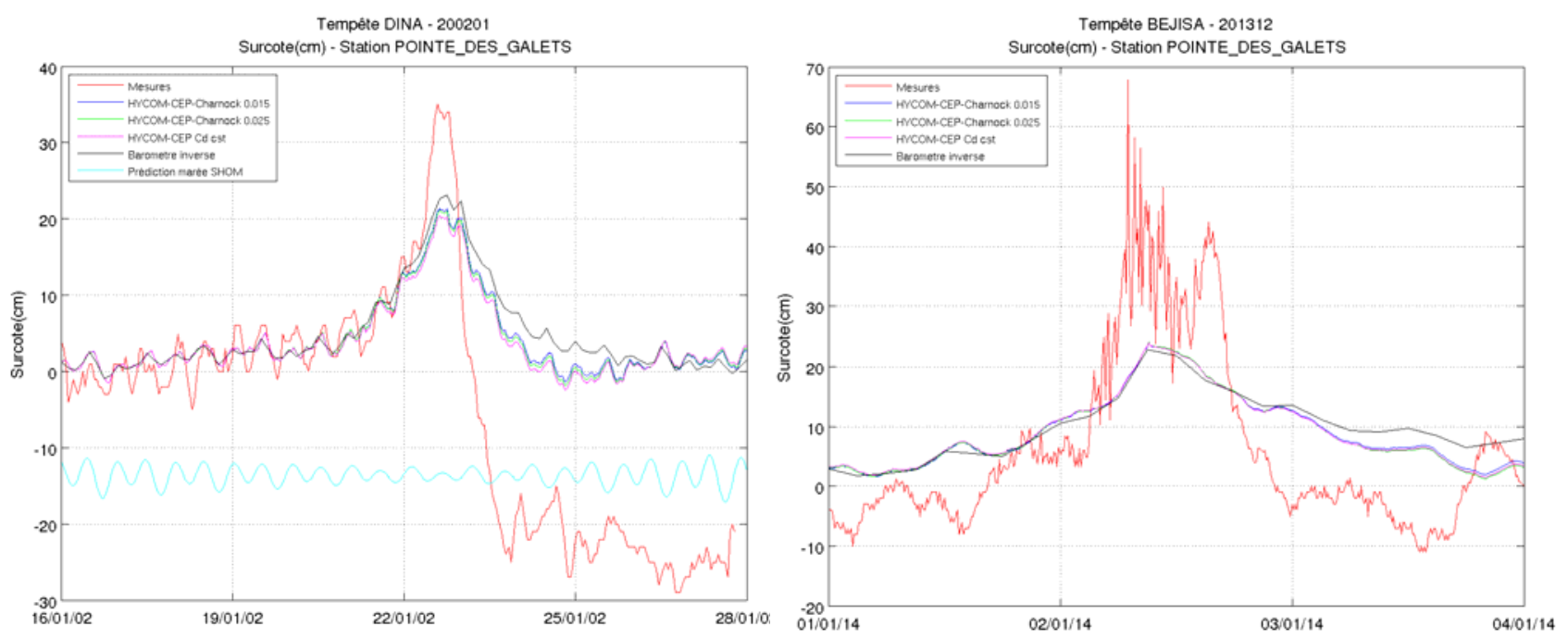

Figure 6. Surcotes observée et modélisées par HYCOM à Pointe des Galets pendant les tempêtes DINA (janv. 2002, à gauche) et BEJISA (janv. 2014, à droite).

\section{Conclusions et perspectives}

La configuration Antilles-Guyane bénéficie d'une carte de friction sur le fond variable, ce qui a permis d'améliorer la représentation de la marée dans tout le domaine par rapport à la version antérieure avec une friction constante. Une paramétrisation du stress de vent avec seuillage a été choisie pour bien reproduire le pic de surcote lors de l'évènement exceptionnel IRMA. 


\section{Thème 1 - Hydrodynamique côtière}

Concernant la configuration Sud-Ouest Océan Indien : La configuration bathymétrique autour des zones d'intérêt comme Mayotte et La Réunion (fortes profondeurs et très forts gradients de bathymétrie) est un facteur qui limite, au moins à cette résolution de calcul, les effets de frottement (en surface et au fond) tels qu'ils sont représentés dans un modèle barotrope. Ceci explique la faible dépendance des résultats de cette configuration aux différentes paramétrisations (frottement sur le fond et stress de vent). Afin d'améliorer cette configuration, la prochaine mise à niveau comportera des descentes d'échelle de modèles avec des grilles et des bathymétries plus résolues (disponibilité de MNT 500 et $100 \mathrm{~m}$ ) qui détailleront certaines remontées bathymétriques et zones de faibles profondeurs autour de Mayotte et La Réunion. La friction au fond sera variable et optimisée par méthode stochastique.

Du fait de l'impact très localisé et de l'intensité des tempêtes, une bonne représentation des forçages météorologiques (trajectoire de la dépression, intensité des forçages) est un des éléments impactant fortement la bonne représentation de la surcote modélisée lors des évènements.

\section{Références bibliographiques}

BARAILLE R., FILATOFF N. (1995). Modèle shallow-water multicouches isopycnal de Miami. Tech. Rep. 003/95, SHOM/CMO.

BOUTET M. (2015). Estimation du frottement sur le fond pour la modélisation de la marée barotrope. Thèse de doctorat, Université de Bretagne Occidentale, Brest, 192 p. https://doi.org/10.13140/RG.2.1.1105.0488

CHARNOCK H. (1955). Wind stress on a water surface. Q.J.R. Meteorol. Soc., Vol. 81, pp 639-640. https://doi.org/10.1002/qi.49708135027

MAKIN V. K. (2005). A note on the drag of the sea surface at hurricane winds. Boundary-Layers Meteorology, Vol. 115, pp169-176. https://doi.org/10.1007/s10546-004-3647-x

POND S., PICKARD G. L. (1998). Introductory dynamical oceanography. Butterworth-Heinmann.

POWELL, M. D., VICKERY, P. J., and REINHOLD, T. A. (2003): Reduced Drag Coefficient for High Wind Speeds in tropical Cyclones, Nature, Vol. 422, pp 279-283. https://doi.org/10.1038/nature01481

BISCARA, L. et al. (2017). Elaboration d'un modèle numérique de terrain (MNT) bathymétrique régional à $500 \mathrm{~m}$ de la zone Antilles-Guyane pour la modélisation hydrodynamique (Projet HOMONIM). Rapport Interne SHOM.

TOLMAN H. L. (2016). Technical note. User manual and system documentation of WAVEWATCH III R. version 5.16. Development Group. Environmental Modeling Center Marine Modeling and Analysis Branch. 